\title{
GPPS-TC-2019-0020
}

\section{DYNAMIC COEFFICIENT IDENTIFICATION FROM EXPERIMENTS: THE EFFECTS OF MEASURING HYDRODYNAMIC FORCES VS. APPLIED FORCES}

\author{
Benstone Schwartz \\ University of Virginia \\ bs9ns@virginia.edu \\ Charlottesville, VA, USA
}

\author{
Roger Fittro \\ University of Virginia \\ rlf9w@virginia.edu \\ Charlottesville, VA, USA
}

\author{
Carl Knospe \\ University of Virginia \\ knospe@virginia.edu \\ Charlottesville, VA, USA
}

\begin{abstract}
A method of evaluating the identification uncertainty in identifying dynamic coefficients of hydrodynamic components, such as fluid-film bearings, seals, and squeezefilm dampers, using test rig data is extended to compare two types of tests: (1) experiments where the applied force is measured and (2) experiments where the hydrodynamic force produced by the test article is measured. Additionally, a comparison is made for each type of test on the sensitivity of the identification uncertainty to assuming the rotor in the test rig is rigid. The results show that validation of dynamic coefficients can be more accurate (i.e. - lower uncertainty) if the hydrodynamic force of the test article is measured.
\end{abstract}

\section{INTRODUCTION}

Industrial rotating machinery such as compressors and gas turbines are trending toward higher operating speed and higher unit load. The more demanding applications need more accurate prediction of system behaviour. Accurate rotordynamic analysis is critical in predicting operating characteristics of systems and ensuring safe, stable operation. Inadequate analysis can lead to millions of dollars in lost productivity or even catastrophic failure. (Gunter \& Weaver, 2016; Rieger, McCloskey, \& Dewey, 1990)

Stability predictions for rotating machines require a rotor model and dynamic properties of system components such as bearings and seals. The dynamic properties of bearings and seals are typically expressed as stiffness and/or damping coefficients. Coefficients are predicted with codes using physics-based models such as a thermoelastohydrodynamic (TEHD) description. (He, 2003)

An API-sponsored survey revealed that dynamic coefficient predictions could vary significantly. (Kocur, Nicholas, \& Lee, 2007) The survey distributed the same bearing, seal, and rotor design to the various groups with rotordynamic modelling capabilities and requested dynamic coefficient predictions and stability predictions. The dynamic coefficient predictions of bearings and seals varied by as much as an order of magnitude for stiffness and damping. For stability predictions, a common eigensolver was used to isolate variation to modelling differences between groups. The stability predictions varied greatly due to the coefficient variations, often predicting a more stable system than the experimental data indicated. One of the conclusions of this survey is that "a gold standard of experimental data is needed for both tilting pad journal bearings and gas labyrinth seal dynamic coefficients."

\section{Experimental Validation of Predictive Models}

Many test rigs have been developed for experimental model validation. (Dimond, Sheth, Allaire, \& He, 2009; Tiwari, Lees, \& Friswell, 2004) These test rigs have two typical forms: 1) the rotor is held rigidly with rolling element bearings and bearing housing motion is excited by a connected actuator 2) the housing is held via a rigid connection to the foundation and the rotor is excited. The first design is more common due to the implementation being simpler. Designs of the second type been implemented with unbalance force providing the excitation. Recently, active magnetic bearings (AMBs) have been used to apply the excitation force to the rotor. Their non-contact nature simplifies the application of force to the rotating shaft.

In a typical dynamic coefficient identification experiment, the applied force on the excited component is measured and the resulting displacement of the excited component relative to the rigidly held component is measured. Using the force and displacement measurement, dynamic coefficients can be calculated.

All measurements of physical quantities, whether directly from a sensor or constructed from the data from several sensors, have some uncertainty directly associated with their measurement principle and its embodiments. Schwartz et al. show that these uncertainties lead to unavoidable uncertainty in the identified dynamic coefficients. (Schwartz, Fittro, \& Knospe, 2017) The identification uncertainty increases as frequency of excitation increases. The uncertainty is also a 
function of the test article's properties. Test articles with smaller stiffness and damping values had higher uncertainty in identified coefficients from experiments. These trends were shown analytically using a simplified model. A tilting-pad journal bearing (TPJB) identification experiment with measurement errors was simulated to see if these trends also appeared in more realistic system models. Measurement errors were simulated with errors varied in a Monte Carlo fashion. These simulation results supported the conclusions developed analytically from the simplified model. This support strongly suggests that the simple models can lead to significant insights into the coefficient identification problem. While this study was targeted towards tilting pad journal bearings, the fundamental conclusions likely apply to the testing of other components as well.

\section{Measuring Hydrodynamic Forces}

Schwartz et al. (2017) analysed identification of dynamic coefficients with two measurements: 1) relative displacements between the excited component (either rotor or housing) and the rigidly held component; and 2) the applied force on the excited component.

One alternative approach to identification is to measure the hydrodynamic force generated by the test article (such as fluid-film bearings, seals, or squeeze-film dampers). Various approaches could be employed to develop such force measurements. For example, forces generated by a squeeze film damper have been constructed by numerically integrating pressure measurements from within the damper. (Feder, Bansal, \& Blanco, 1978) Load cells external to bearing housings have been used to measure bearing force signals. (Chatterton, Pennacchi, Dang, \& Vania, 2015) A new method of measuring hydrodynamic force using acceleration cancellation has been recently proposed for dynamic coefficient identification. (Gancitano, Schwartz, Fittro, \& Knospe, 2018) Identifying dynamic coefficients using hydrodynamic force measurements is less common in the literature than experiments measuring applied force. The increased complexity and cost of hydrodynamic force measurements most likely dissuades investigators from such experiments when no clear and quantifiable advantage have been demonstrated so far. In fact, the effect on uncertainty of using hydrodynamic force measurements rather than applied force measurements has not been previously investigated.

\section{Outline}

In this work, the authors will use the analysis methods previously developed by Schwartz et al. (2017) and compare the identification uncertainty when identifying with applied force measurement to the uncertainty when identifying with hydrodynamic force measurement. First, a single degree-offreedom (SDoF) model is used for an analytic study of the impact of measurement uncertainty. Then a Monte Carlo analysis of this SDoF model is conducted. A higher-fidelity two degrees-of-freedom (2DoF) model is then introduced with a more realistic bearing model and a Monte Carlo analysis is conducted to investigate the effects of measurement uncertainty. Finally, to examine the sensitivity to rigid-rotor modelling assumptions, the lumped-mass rotor model used for truth in the $2 \mathrm{DoF}$ simulation is replaced with a finite-element rotor model (i.e. - flexible) and the effect of rotor flexibility on the identification uncertainty is determined in the case where the identification procedure has assumed the rotor is rigid.

\section{THEORY}

The first analysis simplifies a bearing test rig's dynamics into a SDoF model with representations of the excited housing, rigidly held rotor, and the bearing dynamics. Figure 1 highlights the excited and the rigidly held components. In Figure 2, a simplified representation of the test rig is shown.

While this simplified model is developed for fluid-film bearing experiments, models for other hydrodynamic components could be represented in essentially the same fashion. Therefore, trends seen in this investigation should hold for the experimental investigation of many other hydrodynamic components.

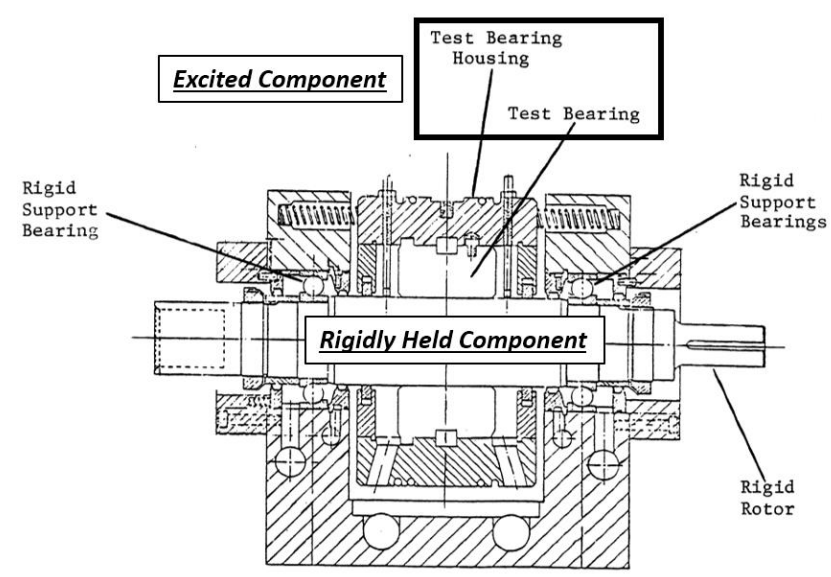

Figure 1 - Fluid-film bearing test rig (cross section) adapted from (Flack, Kostrzewsky, \& Taylor, 1993)

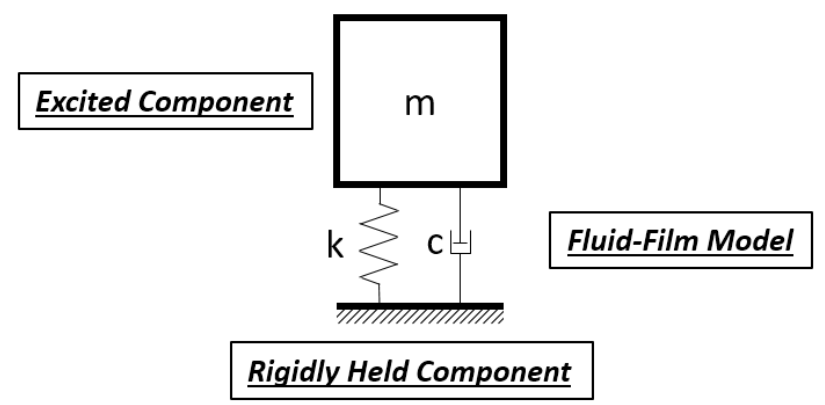

Figure 2 - SDoF Model with components analogous to fluid-film bearing test rig components identified

Following the same analytical approach established by Schwartz et al. (2017), we begin by establishing a frequencydomain representation of the truth model of identification experiment:

$$
\begin{gathered}
\mathrm{F}_{\mathrm{A}}=\left[\left(\mathrm{k}-\mathrm{m} \omega^{2}\right)+j c \omega\right] X \\
\mathrm{~F}_{\mathrm{C}}=[k+j c \omega] X
\end{gathered}
$$

where $F_{A}$ is applied force, $F_{C}$ is hydrodynamic force, and $X$ is the excited component's response to applied forces. 


\section{Modelling Uncertainty}

Systematic measurement error can be modelled as:

$$
\begin{aligned}
& \widehat{F_{A}}(j \omega)=F_{A}(j \omega)\left(1+\Delta_{A}(j \omega)\right) \\
& \widehat{F_{C}}(j \omega)=F_{C}(j \omega)\left(1+\Delta_{C}(j \omega)\right) \\
& \hat{X}(j \omega)=X(j \omega)\left(1+\Delta_{X}(j \omega)\right)
\end{aligned}
$$

where $F_{A}, F_{C}$, and $X$ are the true signals. $\widehat{F_{A}}, \widehat{F_{C}}$, and $\hat{X}$ are the measured signals. $\Delta_{C}, \Delta_{A}$, and $\Delta_{X}$ are complex valued variables representing the errors in magnitude and phase of each measured signal relative to the corresponding true signal. To identify dynamic coefficients, one seeks to find coefficient estimates $\hat{k}$ and $\hat{c}$ that agree with these measured signals. To achieve this agreement, the identified coefficients should satisfy the dynamics of the truth model when the measured signals are used. Specifically:

$$
\begin{gathered}
\widehat{F_{A}}(j \omega)=\left[\left(\hat{k}-m \omega^{2}\right)+j \hat{c} \omega\right] \hat{X}(j \omega) \\
\widehat{F_{C}}(j \omega)=[\hat{k}+j \hat{c} \omega] \hat{X}(j \omega)
\end{gathered}
$$

Because the measurements have error, the identified coefficients will be different from the true values. In the following analysis, it is assumed that mass is known perfectly. However, the same approach can be used with uncertain inertia also identified from data.

For small values of error $\left(\left|\Delta_{X}\right|,\left|\Delta_{A}\right|,\left|\Delta_{C}\right| \ll 1\right)$, two useful simplifications may be employed:

$$
\begin{aligned}
& \frac{1+\Delta_{X}}{1+\Delta_{A}} \approx 1+\Delta_{X}+\Delta_{A}=1+\Delta_{X A} \\
& \frac{1+\Delta_{X}}{1+\Delta_{C}} \approx 1+\Delta_{X}+\Delta_{C}=1+\Delta_{X C}
\end{aligned}
$$

Combining Equations 1 through 8 and simplifying yields:

$$
\begin{gathered}
\left(1+\Delta_{t o t}\right)\left[\left(k-m \omega^{2}\right)+j c \omega\right]=\left(\hat{k}-m \omega^{2}\right)+j \hat{c} \omega \\
\left(1+\Delta_{t o t}\right)[k+j c \omega]=\hat{k}+j \hat{c} \omega
\end{gathered}
$$

Separating these equations into real and imaginary parts and rewriting them in terms of percent errors in the identified dynamic coefficients provides the following relationships for the case where the applied force is measured:

$$
\begin{gathered}
\frac{\hat{k}-k}{k}=\operatorname{Re}\left(\Delta_{X A}\right)\left(1-\frac{m \omega^{2}}{k}\right)-\operatorname{Im}\left(\Delta_{X A}\right)\left(\frac{c \omega}{k}\right) \\
\frac{\hat{c}-c}{c}=\operatorname{Re}\left(\Delta_{X A}\right)+\operatorname{Im}\left(\Delta_{X A}\right)\left(\frac{k}{c \omega}-\frac{m \omega}{c}\right)
\end{gathered}
$$

Similar expressions can be derived for the case where hydrodynamic force is measured:

$$
\begin{aligned}
& \frac{\hat{k}-k}{k}=\operatorname{Re}\left(\Delta_{X C}\right)-\operatorname{Im}\left(\Delta_{X C}\right)\left(\frac{c \omega}{k}\right) \\
& \frac{\hat{c}-c}{c}=\operatorname{Re}\left(\Delta_{X C}\right)+\operatorname{Im}\left(\Delta_{X C}\right)\left(\frac{k}{c \omega}\right)
\end{aligned}
$$

For the SDoF model of a test rig, Equations 11-14 provide the relationships that described how systematic measurement errors affect the accuracy of identified dynamic coefficients. The actual measurement errors are unknown in an experiment. However, bounds on measurement errors can be quantified through data provided by sensor vendors. Data sheets for commercial sensors often contain calibration data for the sensor or other characterizations of typical error. Using the uncertainty bounds for each measured signal, an analysis allows the range of possible identified coefficients to be determined. This range is then the uncertainty in identifying dynamic coefficients from the measurements used.

\section{METHODOLOGY}

\section{Monte Carlo Analysis of Uncertainty of SDoF Model}

In contrast to the analytical approach to evaluating how measurement uncertainty propagates to identification uncertainty, simulations of the SDoF freedom can be performed. Simulations allow the testing of a number of measurement uncertainty combinations to determine the resulting uncertainty in coefficient identification. Using this approach the simplifications used in Equations $8 \mathrm{a}$ and $8 \mathrm{~b}$ are no longer necessary. In each simulation a single-frequency identification method is used to generate coefficient estimates for that test frequency. Combining many simulations together allows the determination of coefficient uncertainty as a function of test frequency.

A block diagram of the model used is shown in Figure 3. The truth model simulation produces three physical signals: the applied force on the excited component, the resulting displacement, and the hydrodynamic force. Multiplicative error is then applied to these signals to produce the measured signals of the simulation. The complex-valued multiplicative error is sampled from a region of the complex plane defined by a magnitude uncertainty bound and a phase uncertainty bound, as shown in Figure 4. The error bounds specified for this analysis are presented in Table 1. After a sufficient number of simulations were conducted for a particular excitation frequency, estimates of the maximum and minimum of identified coefficients for that frequency were found.

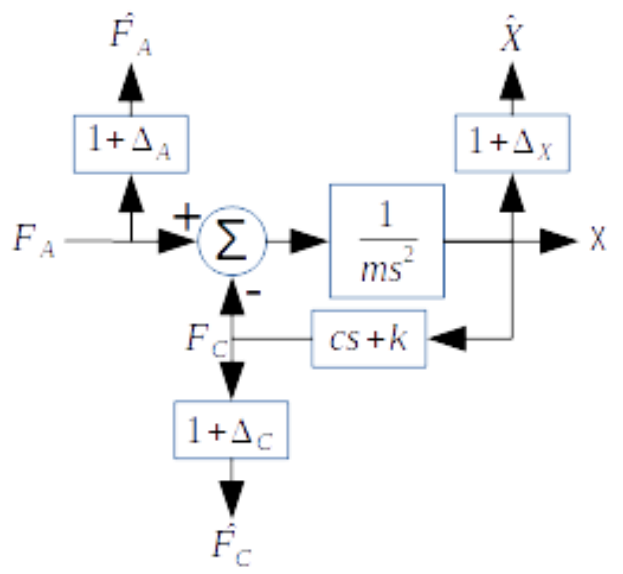

Figure 3 - Block Diagram of SDoF Model Used in Monte Carlo Study

PARAMETER

\begin{tabular}{c|c}
\hline DISPLACEMENT MAGNITUDE & $1 \%$ \\
DISPLACEMENT PHASE & $1^{\circ}$ \\
FORCE MAGNITUDE & $1 \%$ \\
FORCE PHASE & $1^{\circ}$ \\
Table 1 - Uncertainty Bound Specification for the \\
Measured Signals in the Analysis
\end{tabular}




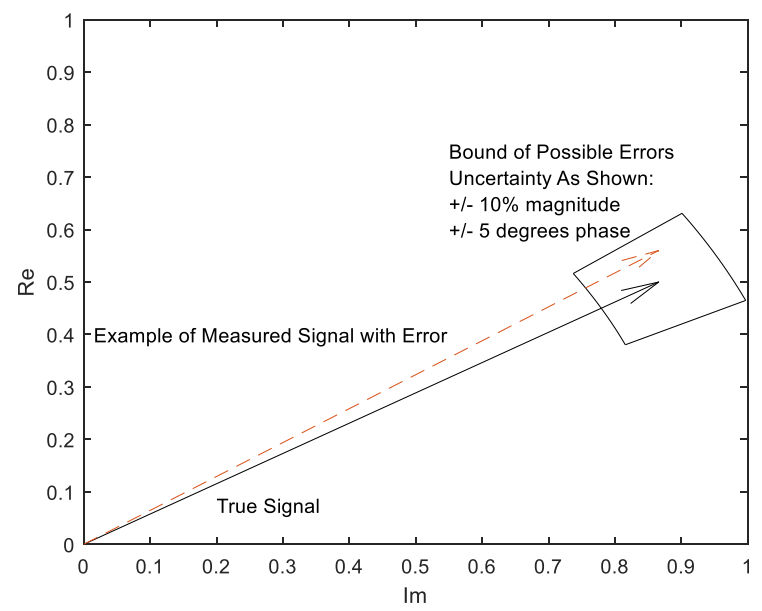

Figure 4 - Error Region Possible for Signal

\section{Higher Fidelity Simulation}

In addition to the SDoF model, a two-degree of freedom model was also analyzed. In this model the excited component has two orthogonal forces - horizontal and vertical - applied at the same test frequency. Two linearly independent sets of applied forces are used for coefficient identification. The applied forces result in relative displacement between the excited component and stationary component. In response, hydrodynamic forces are generated according to the prescribed bearing truth model. These forces then act in an equal and opposite fashion on the excited component and on stationary component. Two orthogonal sensors are modelled on either side of the fluid-film component housing. In the simulation model these displacement sensor signals are averaged to produce a single displacement measurement for each orthogonal plane. Figure 5 shows a high-level schematic of the dynamics of the $2 \mathrm{DoF}$ model.

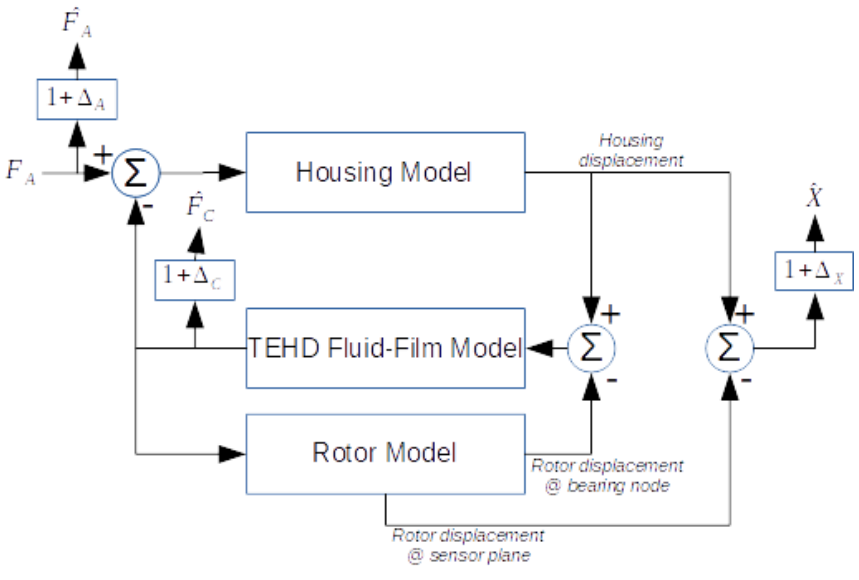

Figure 5 - Schematic for Higher-Fidelity Simulation

Two cases will be analysed. In each case, the model is based on the fluid film bearing test rig described in Flack et al. (1993) In this test rig, the excited component is the housing and the rotor is rigidly held (see Figure 2). A TPJB model generated from a TEHD bearing code was used as the bearing truth model. The bearing model parameters for this study are the same as those found in the Schwartz et al. (2017) study.
The dynamic coefficients of the bearing from TEHD analysis are presented in Figures 6 and 7 for reference.

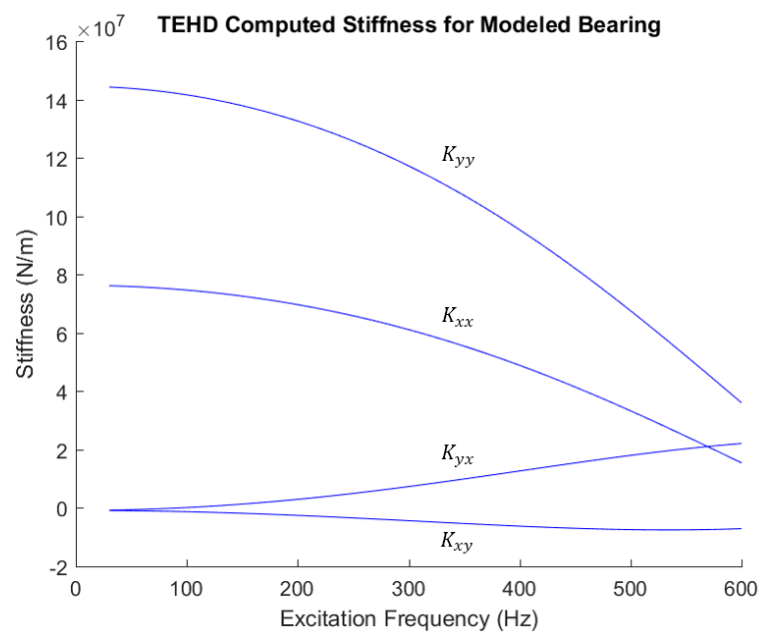

Figure 6 - Stiffnesses from TEHD Bearing Model

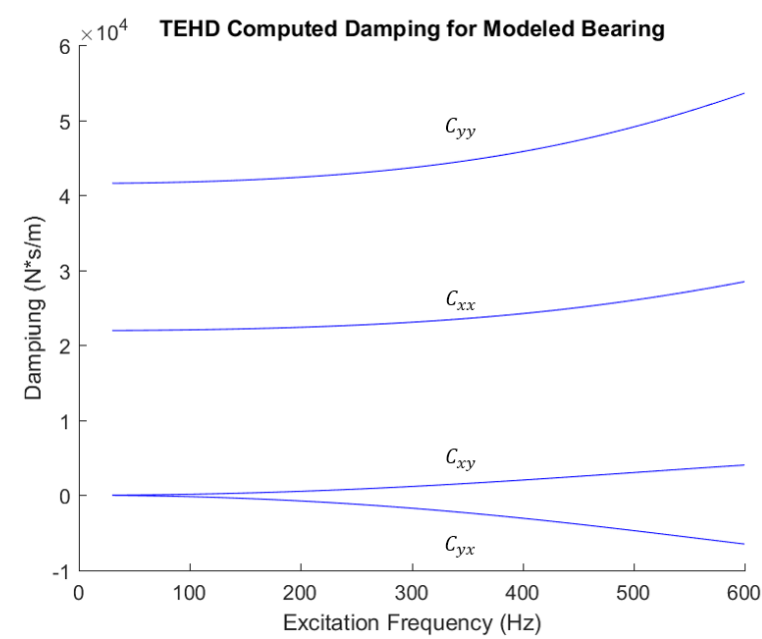

Figure 7 - Damping from TEHD Bearing Model

\section{Truth Model 1}

The first analysis is performed with the housing and rotor completely rigid. In reality there are other dynamics that will be involved. For example, in the Flack et al. (1993) test rig supports are provided to prevent rotation and axial motion of the housing. In the simulation, the housing is modelled as ideal: the only forces acting upon it are the applied force and the hydrodynamic bearing force from the TPJB. Although the two sensor planes included for sensors attached on either side of the bearing housing, the rigid rotor model means there is no difference between the displacements at the sensor planes and the displacements at the fluid-film bearing's node.

\section{Truth Model 2}

In the second case examined, the rotor truth model used is obtained from finite-element analysis. In this model, the displacement at each of the various stations along the rotor responds in a unique fashion to the applied force as governed by the rotordynamics. Thus, the displacements at the sensor 
locations will not match the relative displacement between the bearing journal and housing.

\section{Identification Model}

Bearing dynamic coefficients were identified using both Truth Model 1 and Truth Model 2 through simulation. The identification was performed in two ways for each truth model: with displacement measurements and 1) applied force measurements or 2) hydrodynamic force measurements. The identification model in all simulations assumes that the housing and rotor are rigid. The treatment of these components as rigid is standard practice in previous investigations (i.e, the components are deemed "rigid enough" to ignore flexibility). Thus, Truth Model 1 and the Identification Model have an identical dynamic structure, differing only in that the Identification Model uses data affected by multiplicative measurement errors. Since rotor flexibility is included in Truth Model 2, it differs from the Identification Model also in its dynamics. Thus, comparison of Truth Model 1 and 2 results allows the impact of the "rigid enough" assumption to be quantified. The 2DoF identification models are:

$$
\begin{gathered}
\boldsymbol{F}_{\boldsymbol{A}}=\left[\begin{array}{ll}
H_{x x}(j \omega) & H_{x y}(j \omega) \\
H_{y x}(j \omega) & H_{y y}(j \omega)
\end{array}\right] \boldsymbol{X} \\
H_{x x}(j \omega)=\left(K_{x x}(j \omega)-m \omega^{2}\right)+j \omega C_{x x}(j \omega) \\
H_{x y}(j \omega)=K_{x y}(j \omega)+j \omega C_{x y}(j \omega) \\
H_{y x}(j \omega)=K_{y x}(j \omega)+j \omega C_{y x}(j \omega) \\
H_{y y}(j \omega)=\left(K_{y y}(j \omega)-m \omega^{2}\right)+j \omega C_{y y}(j \omega) \\
\boldsymbol{F}_{\boldsymbol{C}}=\left[\begin{array}{ll}
K_{x x}(j \omega)+j \omega C_{x x}(j \omega) & K_{x y}(j \omega)+j \omega C_{x y}(j \omega) \\
K_{y x}(j \omega)+j \omega C_{y x}(j \omega) & K_{y y}(j \omega)+j \omega C_{y y}(j \omega)
\end{array}\right] \boldsymbol{X}
\end{gathered}
$$

where Equation 15 represents the identification model for the case in which applied force is measured and Equation 16 represents the identification model for the case in which hydrodynamic force is measured. $\boldsymbol{F}_{\boldsymbol{A}}$ and $\boldsymbol{F}_{\boldsymbol{C}}$ are vectors of forces in the horizontal $(x)$ and vertical $(y)$ directions. $\boldsymbol{X}$ is the vector of horizontal and vertical relative displacement between the excited component and the stationary component.

\section{Monte Carlo Analysis Setup}

As with the SDoF model, the measurement errors will be sampled uniformly within the range defined in Table 1. For the higher fidelity model, each axis of each plane of displacement measurement has an independent error applied. The force measurements have error applied on each axis independently.

\section{RESULTS AND DISCUSSION}

\section{SDoF Results}

Figure 8 highlights the maximum and minimum of identified stiffness in the SDoF model. Applied force measurement results and hydrodynamic force measurement results are shown. The range between maximum and minimum can be found and compared with the true value to find an uncertainty magnitude. The uncertainty magnitudes of identified coefficients in the SDoF analysis are summarized in Figure 9. Figure 8 and 9 are presented with test frequencies from $0 \mathrm{~Hz}$ to $1000 \mathrm{~Hz}$ to highlight the difference in trend for a different selection of force signal in identification. The remaining figures focus on $0 \mathrm{~Hz}$ to $500 \mathrm{~Hz}$ as the differences in trends are evident in the range plotted.

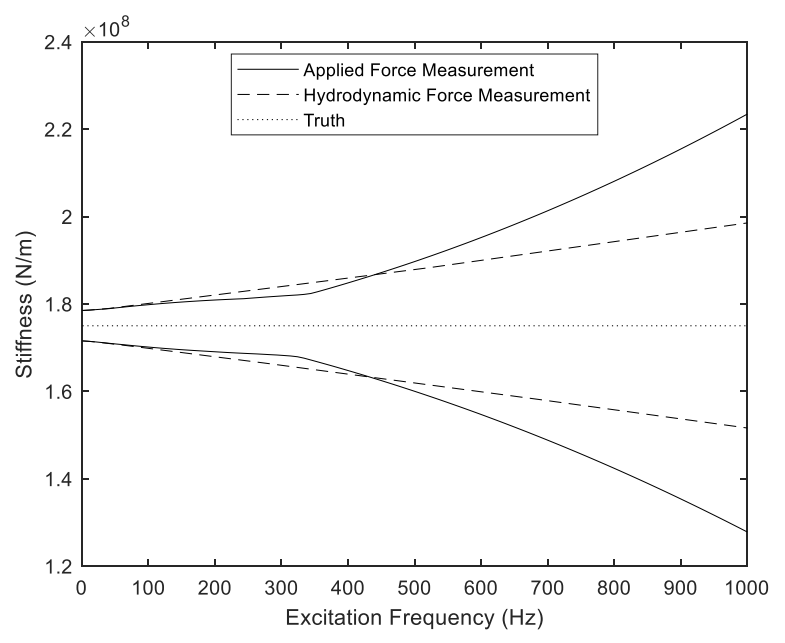
Figure 8 - Minimum and Maximum of Identified
Bearing Stiffness for SDoF Simulation

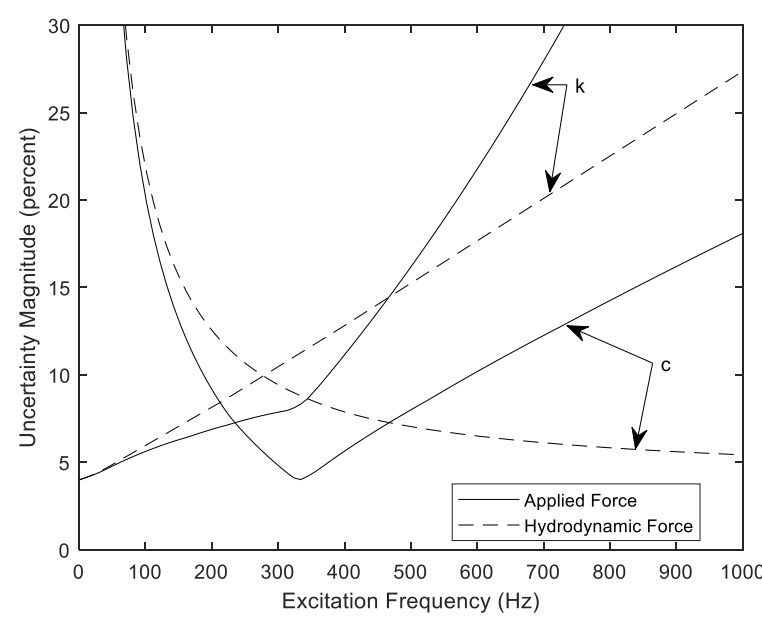

Figure 9 - SDoF Simulation Uncertainty Magnitudes for Bearing Stiffness and Damping Coefficients. Comparison of Identification Using Applied Force Versus Using Hydrodynamic Force Measurements

\section{Higher Fidelity Results}

For the TPJB identification experiment simulation, crosscoupled stiffness and cross-coupled damping results are not presented because for TPJBs the cross-coupled forces are not significant contributors to dynamics. (He, Byrne, Cloud, \& Vazquez, 2016) For a different component such as a seal, the cross-coupled results can be analysed.

Figure 10 summarizes uncertainty magnitudes for identified $\mathrm{K}_{\mathrm{xx}}$ and $\mathrm{K}_{\mathrm{yy}}$ for Case 1 of the higher fidelity simulation. Figure 10 summarizes uncertainty magnitudes for identified $\mathrm{C}_{\mathrm{xx}}$ and $\mathrm{C}_{\mathrm{yy}}$ for Case 1 of the higher fidelity simulation. Both applied force measurement and 
hydrodynamic force measurement cases are presented for comparison.

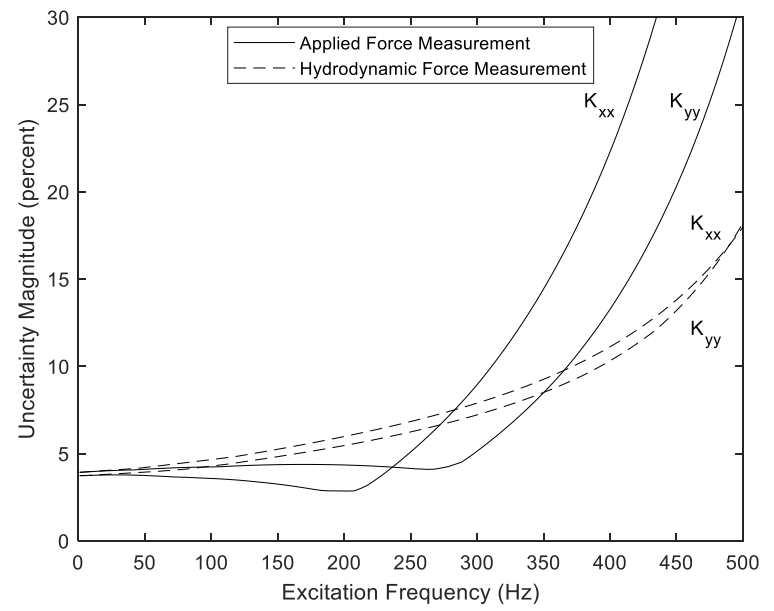

Figure 10 - Uncertainty Magnitude for Identified $\mathrm{K}_{\mathrm{xx}}$ $\mathrm{K}_{\mathrm{yy}}$. Comparison of Results using Applied Force and Hydrodynamic force measurements

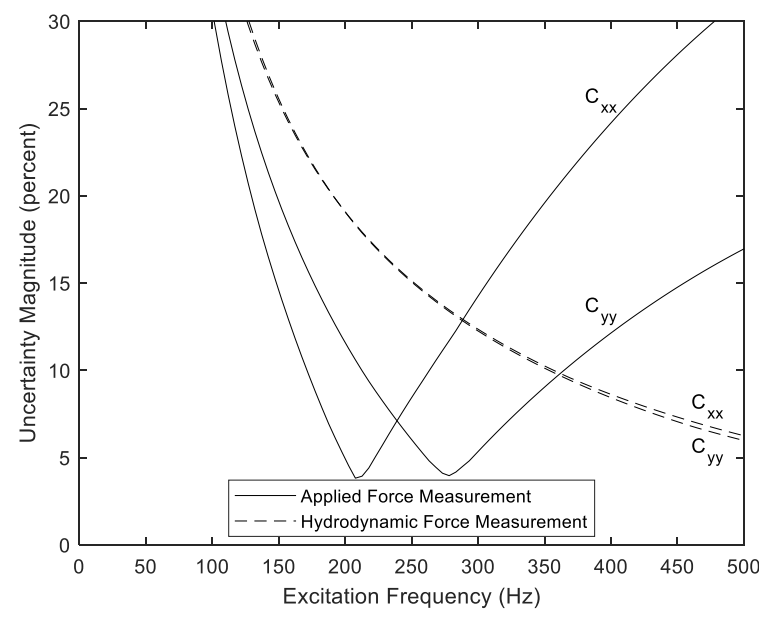

Figure 11 - Uncertainty Magnitude for Identified $\mathbf{C}_{\mathrm{xx}}$ $\mathrm{C}_{\mathrm{yy}}$. Comparison of Results using Applied Force and Hydrodynamic force measurements

Figures 12 and 13 present comparisons between Case 1 where the truth model has a rigid rotor and Case 2 where the rotor is not assumed to be rigid. Figure 12 compares $K_{x x}$ identification uncertainty magnitude when coefficients are identified with applied force measurement. Figure 13 compares the uncertainty magnitude when coefficients are identified with hydrodynamic force measurement.

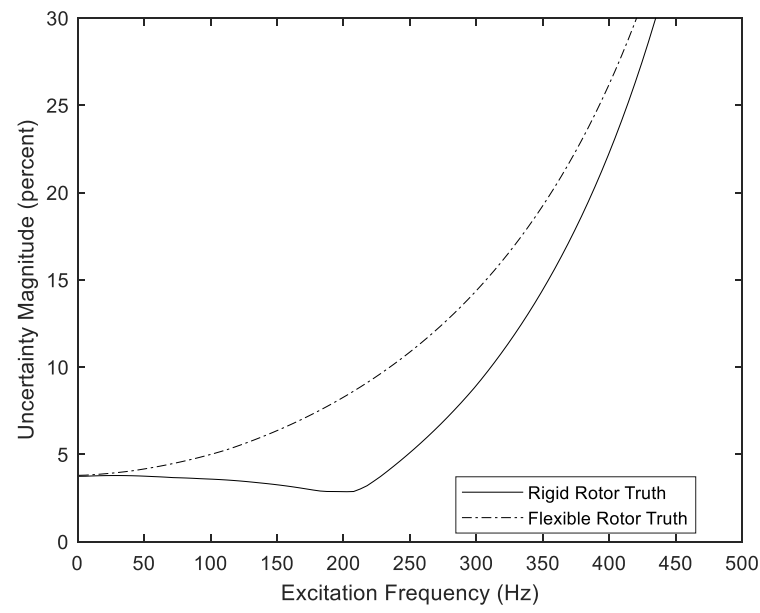

Figure 12 - Uncertainty Magnitude for Identified $\mathrm{K}_{\mathrm{xx}}$. Comparison of Results Using Applied Force Measurements When Truth Model has Rigid Rotor and Truth Model has Flexible Rotor

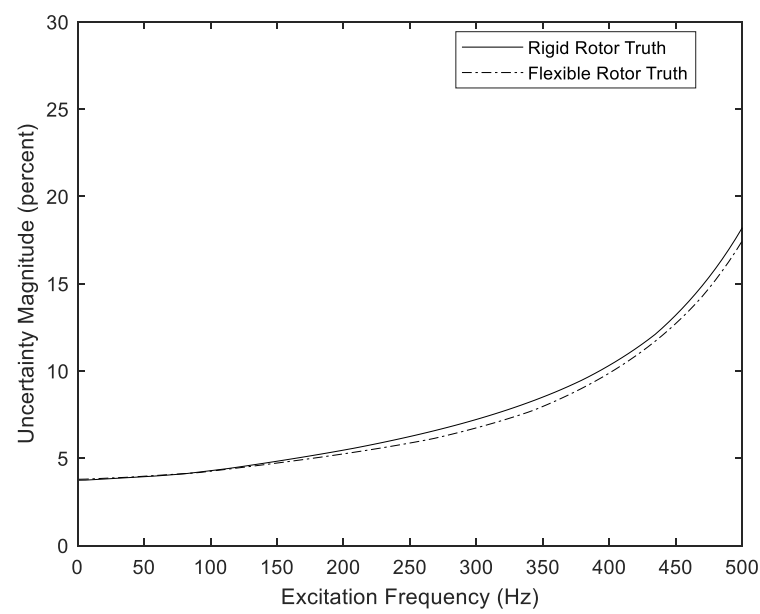

Figure 13 - Uncertainty Magnitude for Identified $\mathrm{K}_{\mathrm{xx}}$. Comparison of Results Using Hydrodynamic Force Measurements When Truth Model has Rigid Rotor and Truth Model has Flexible Rotor

\section{Discussion}

The SDoF simulation results show that the ultimate growth trend of uncertainty for both stiffness and damping is greater when measuring applied force than when measuring hydrodynamic force. There is a small region where measuring applied force has lower uncertainty. This is around the critical frequency of the system where the dynamics is more sensitive to the component properties. However, outside of that range, applied force measurement has a quadratic uncertainty trend as a function of test frequency while measuring hydrodynamic force has a linear uncertainty trend with respect to test frequency.

The higher-fidelity simulation demonstrates the same basic trends seen in the SDoF simulation. As test frequency gets higher, measuring hydrodynamic force results in lower dynamic coefficient identification uncertainty. Furthermore, the uncertainty was greater for $\mathrm{K}_{\mathrm{xx}}$ and $\mathrm{C}_{\mathrm{xx}}$ compared to $\mathrm{K}_{\mathrm{yy}}$ 
and $\mathrm{C}_{\mathrm{yy}}$. This is because the vertical principal coefficients are larger in magnitude than the horizontal principal coefficients. Equations 11-14 show this behaviour. The true damping and true stiffness are in the denominator and therefore if the true properties become smaller in amplitude, the uncertainty magnitude increases. Identifying with measurement of applied force is more sensitive to changes in the truth's magnitude. This suggests that measuring hydrodynamic force is more applicable to a broader range of components. Foil bearings, for example, would have much lower stiffness than other types of bearings and identification experiments for foil bearing coefficients would benefit from measuring the hydrodynamic bearing force.

When comparing Case 1 and Case 2 where the major difference is the rotor truth model being ideally rigid or allowed to bend, the increase in uncertainty is noticeable with identification using applied force measurement. When the hydrodynamic bearing force is measured, the difference between Case 1 and Case 2 is much smaller. The rotor model in the simulation is of a rotor that has been considered "rigid enough" to assume ideally rigid in past investigations. This indicates that in general, not modelling a rotor's dynamics even if it is very rigid leads to additional uncertainty. The larger implication could be that un-modelled dynamics such as substructure dynamics, even if the substructure is very rigid compared to component properties, need to be adequately modelled to reduce uncertainty. It is also important to note that measuring hydrodynamic force in the simulation was less sensitive to un-modelled dynamics.

One uncertainty effect for Case 2 that uncertainty magnitudes presented don't capture is an offset in identified coefficients. While the SDoF analysis and higher-fidelity analysis of Case 1 had the range of identified coefficients cantered about the true value, the analysis of Case 2 resulted in the centre of the range of identified coefficients being offset from truth.

The higher-fidelity analysis results support the conclusions the simplified, analytical model would suggest. This suggests that while uncertainty predictions for an experiment needs careful modelling of the experimental apparatus, some design choices such as which force signal to measure can be evaluated using a simple model.

\section{Reasons for the Difference in Uncertainty}

The root cause of the differences in uncertainty can be understood by looking at Equations 11 and 12 for identifying $k$ and $c$ with applied force measurements and Equations 13 and 14 for identifying $k$ and $c$ with hydrodynamic force measurements. In Equations 11 and 12, there are terms with the excited component's inertia, $m$. The implication here is that the applied force measurement associated with a specific measured displacement response has a part associated with the excited component's inertia and a part associated with the hydrodynamic force applied on the excited component. The force measurement error, then, is applied to the sum of both parts. When the hydrodynamic force is measured directly, there is no inertial part to the measurement. Thus the measurement error is only applied to the hydrodynamic force.
As a result, the force error with applied force measurement is greater leading to greater uncertainties.

When comparing the case with a rigid rotor truth model with a flexible rotor truth model, the reason for the larger difference when identifying with applied force measurement comes from the inertia again. The dynamical difference between sensor planes and bearing node in the rotor dynamics is amplified when inertial forces are involved in the dynamics. Because identifying with hydrodynamic force measurements does not require knowledge of the inertia in the analysed models, the effect of a flexible rotor when identified as a rigid rotor is amaller.

\section{CONCLUSIONS}

A simulation was performed to compare the effect on dynamic coefficient identification uncertainty based on measuring applied force on a test rig versus measuring the hydrodynamic force.

- Measuring hydrodynamic forces may be more accurate (i.e. - lower uncertainty) in identifying dynamic coefficients. If the measurement of applied force and the measurement of hydrodynamic force have the same error bounds, then measuring hydrodynamic force is more accurate. For cases in which they are not the same, the analysis presented in this work can be adapted to compare different error bounds.

- Measuring hydrodynamic forces for identifying dynamic coefficients may be less sensitive to un-modelled dynamics. In the analyses presented here, the uncertainty only changed slightly when the rotor truth was not ideal even though the identification model did not account for that.

- Uncertainty analysis is important to determining the ability of test rigs to identify dynamic coefficients. In addition to evaluating existing test rigs, the methods described in this work can be utilized to make design decisions regarding uncertainty for new test rig construction.

- The numerical values presented in this work may not be representative for active test rigs because of the assumptions made. However, the relative effects are still valid. Numerical estimates for a specific application may be obtained by updating the values of the measurement uncertainty bounds, housing model, and rotor model in the analysis.

Engineers and researchers should perform a careful uncertainty analysis of identification experiments to understand how accurately coefficients can be identified.

\section{Future Work}

In this work, simulations were used to compare two methods of identifying hydrodynamic coefficients. The measurement error specifications between the two methods were kept consistent to provide an apples-to-apples comparison. Another way to apply the uncertainty analysis method presented in this work is to incorporate data from real test rigs. For example, the experimental method and instrumentation from published literature can be modelled. 
Data about the force sensors, displacement measurement sensors, and other sensors in the test rigs can be fed into an uncertainty analysis like the one performed in this work. This will estimate the uncertainty of coefficient identification from test rigs used in published literature. The uncertainty estimated with this method can be used to inform researchers on the accuracy of published data. The results of this uncertainty analysis will show greater uncertainty in estimated dynamic coefficients compared with uncertainty analyses presented in published literature if the published literature did not account for systematic errors present in the system.

\section{NOMENCLATURE}

$\omega$ - excitation frequency for identification experiment

$m$ - mass of component that excitation force is applied to

$k$ - true stiffness of test article in SDoF analytic study

$c$ - true damping of test article in SDoF analytic study

$X$ - true response signal (displacement)

$F_{A}$ - true applied force signal

$F_{C}$ - true hydrodynamic force signal

$\widehat{k}$ - identified stiffness of test article in SDoF analytic study

$\hat{C}$ - identified damping of test article in SDoF analytic study

$\hat{X}$ - measured response signal (displacement)

$\widehat{F_{A}}$ - measured applied force signal

$\widehat{F_{C}}$ - measured hydrodynamic force signal

$\Delta_{X}$ - displacement measurement error

$\Delta_{A}$ - applied force measurement error

$\Delta_{C}$ - hydrodynamic force measurement error

\section{ACKNOWLEDGMENTS}

This work was funded by the Rotating Machinery and Controls (ROMAC) Laboratories at the University of Virginia.

Many thanks to Eric Maslen who offered invaluable insight to this work.

\section{REFERENCES}

Chatterton, S., Pennacchi, P., Dang, P. V., \& Vania, A. (2015). Identification of Dynamic Force Coefficients of a FivePat Tilting-Pad Journal Bearing, 21, 931-941. https://doi.org/10.1007/978-3-319-06590-8

Dimond, T. W., Sheth, P. N., Allaire, P. E., \& He, M. (2009). Identification Methods and Test Results for Tilting Pad and Fixed Geometry Journal Bearing Dynamic Coefficients - A Review. ROMAC Report, 16(525), 1343. https://doi.org/10.3233/SAV-2009-0452

Feder, E., Bansal, P. N., \& Blanco, A. (1978). Investigation of Squeeze Film Damper Forces Produced by Circular Centered Orbits. Journal of Engineering for Power, 100(1), 15. https://doi.org/10.1115/1.3446313

Flack, R. D., Kostrzewsky, G. J., \& Taylor, D. V. (1993). A hydrodynamic journal bearing test rig with dynamic measurement capabilities. Tribology Transactions, $36(4)$, 497-509. https://doi.org/10.1080/10402009308983190

Gancitano, P., Schwartz, B., Fittro, R., \& Knospe, C. (2018). Development of an Active Load Cell Force Measurement Test Rig. In 16th International
Symposium on Magnetic Bearings. Beijing, China.

Gunter, E. J., \& Weaver, B. (2016). Kaybob Revisited: What We Have Learned About Compressor Stability from Self-Excited Whirling. Advances in Acoustics and Vibration, 1-22.

He, M. (2003). Thermoelastohydrodynamic Analysis of a Fluid Film Journal Bearing. University of Virginia.

He, M., Byrne, J. M., Cloud, C. H., \& Vazquez, J. A. (2016). Fundamentals of fluid film journal bearing operation and modeling. Proceedings of Asia Turbomachinery \& Pump Symposium. Retrieved from https://oaktrust.library.tamu.edu/handle/1969.1/160284

Kocur, J. A., Nicholas, J. C., \& Lee, C. C. (2007). Surveying Tilting Pad Journal Bearing and Gas Labyrinth Seal Coefficients and Their Effect on Rotor Stability. In Proceedings of the Thirty-Sixth Turbomachinery Symposium (p. 10). Retrieved from https://oaktrust.library.tamu.edu/bitstream/handle/1969 $.1 / 163145 /$ ch02-kocur.pdf?sequence $=1 \&$ is Allowed=y

Rieger, N. F., McCloskey, T. H., \& Dewey, R. P. (1990). The high cost of failure of rotating equipment. Proceedings of the 44th Meeting of the Mechanical Failures Prevention Group, 3-19.

Schwartz, B., Fittro, R., \& Knospe, C. (2017). Understanding the effect of systematic errors on the accuracy of experimental measurements of fluid-film bearing dynamic coefficients. In Proceedings of the ASME Turbo Expo (Vol. https://doi.org/10.1115/GT201764665

Tiwari, R., Lees, A. W., \& Friswell, M. I. (2004). Identification of dynamic bearing parameters: A review. Shock and Vibration Digest, 36(2), 99-124. https://doi.org/10.1177/0583102404040173 